\title{
REGULAR HJELMSLEV PLANES. II
}

\author{
BY \\ DIETER JUNGNICKEL ${ }^{1}$
}

\begin{abstract}
In this paper we introduce the notion of an $x$-partition for a Hjelmslev-matrix ( $H$-matrix). This allows us to prove a new composition theorem for $H$-matrices. We obtain the existence of $(t, r)$ - $H$-matrices and hence of regular $(t, r)$ - $H$-planes for infinitely many series of invariants which were not yet known.

In fact, many of these invariants were not even known to occur as the invariants of any $H$-plane at all (whether regular or not).
\end{abstract}

0. Introduction. In 1976, the author introduced the notion of a 'regular' projective Hjelmslev plane (briefly: regular $H$-plane) [4]; such $H$-planes admit a nice abelian collineation group. We proved that there are regular $H$-planes with invariants $\left(q^{n}, q\right)$ for every prime power $q$. We could also show that there are regular $(t, r)-H$-planes (where $t$ is not a power of $r$ ) for all the invariant pairs of $H$-planes that had been constructed by Drake and Lenz in [3]. Finally, we could also obtain some invariant pairs $(t, r)$, that previously were not even known to be the invariants of an $\mathrm{H}$-plane at all (whether regular or not).

To prove these results, the notions of $H$-matrices and $x$-choices for $H$ matrices were essential, allowing certain composition methods. In this paper we want to introduce the notion of an $x$-partition for an $H$-matrix; we will then prove that certain $\left(q^{n}, q\right)-H$-matrices possess $\left(q^{n-1}+1\right)$-partitions. We prove a composition theorem using this notion; this will enable us to obtain regular $(t, r)-H$-planes for many new invariant pairs. In particular, we will be able to obtain many of the invariant pairs $(t, r)$ constructed in [2]; but we will also obtain infinitely many pairs totally unknown up to now. These results will be improved in the last section by constructing $\left(q^{n}, q\right)-H$-matrices with even better partitions.

1. Preliminary knowledge. We refer the reader to the literature for the definition of projective Hjelmslev planes ( $H$-planes) and for the invariants of

Received by the editors December 1, 1976 and, in revised form, March 21, 1977.

AMS (MOS) subject classifications (1970). Primary 05B25, 05B10; Secondary 50A99, 50D99.

Key words and phrases. Hjelmslev plane, regular Hjelmslev plane, Hjelmslev-matrix

'The author acknowledges the hospitality of the University of Florida during the time of this research. 
such planes (we only consider the finite case here); cf. e.g. [3], [4]. Regarding the general geometric and combinatorial background, the reader should consult e.g. [1]. The definition of a regular $H$-plane is in [4, 2.1]. For the convenience of the reader, we recall the definition of a $(t, r)$ - $H$-matrix:

1.1 Definition [4]. Let $N$ be an abelian group of order $t^{2}$ and $A:=\left(a_{i k}\right)$ $(i=0, \ldots, r ; k=1, \ldots, t)$ be a matrix with entries from $N . A$ is called a $(t, r)-K$-matrix if

(1.1) for each pair $(i, j)$ with $i \neq j$ and $i, j \in\{0, \ldots, r\}$, we have $\left\{a_{i k}-\right.$ $\left.a_{j m}: k, m=1, \ldots, t\right\}=N$

and a $(t, r)$-H-matrix if furthermore

(1.2) the $(r+1) t(t-1)$ differences $a_{i k}-a_{i m}(i=0, \ldots, r ; k, m=$ $1, \ldots, t)$ contain each nonzero element of $N$ at least twice. ${ }^{2}$

1.2 THEOREM. $A$ regular $H$-plane with invariants $(t, r)$, where $r$ is a prime power, exists iff there is $a(t, r)-H$-matrix.

Proof. [4, 2.8].

1.3 THEOREM. An $(r, r)$ - $K$-matrix is in fact an $H$-matrix and $r$ is a prime power. Furthermore, each row of the matrix is a subgroup of $N$.

Proof. [4, 5.7] and [5, 3.1 and the proof of 3.1].

1.4 Definition [5]. Let $A$ be a $(t, r)-H$-matrix over $N$. $A$ is called balanced of type $n$ if there exist subgroups $E=U_{n}<U_{n-1}<\cdots<U_{2}<U_{1}=N$ satisfying

(1.3) there are numbers $\lambda_{i}(i=1, \ldots, n-1)$ such that $x \in U_{i} \backslash U_{i+1}$ iff $x$ occurs precisely $\lambda_{i}$ times as a difference from $A$ (according to (1.2));

(1.4) there are numbers $q_{2}, \ldots, q_{n}$ such that the order of $U_{i}$ is $\left(q_{n} \cdots q_{i+1}\right)^{2}$ (note that $t=q_{2} \cdots q_{n}$ );

(1.5) for all $i=1, \ldots, n-1 ; j=0, \ldots, r ; k=1, \ldots, t$, the following holds:

$$
\left|T_{j k}^{i}\right|=\left|\left\{a_{j m}: a_{j m} \equiv a_{j k} \bmod U_{i}\right\}\right|=q_{n} q_{n-1} \cdots q_{i+1} .
$$

The $q_{i}$ are called step parameters, the $\lambda_{i}$ intersection numbers. $t_{i}$ is defined by $t_{i}:=q_{2} q_{3} \cdots q_{i}(i=2, \ldots, n-1)$.

1.5 THEOREM. An $\left(r^{n-1}, r\right)$-H-matrix that is balanced of type $n$ exists iff $r$ is a prime power. In this case, all step parameters are equal to $r$ and we have $\lambda_{i}=r^{i}(i=1, \ldots, n-1)$.

Proof. [5, 2.15 and 3.5], using [6, 1.1], [5, 2.12 and the proof of 2.12] and finally $[5,2.10]$.

\footnotetext{
${ }^{2} \mathrm{By}$ adding $-a_{i 1}$ to row $i$ of $A$ for $i=0, \ldots, r$ we obtain a $(t, r)-K$-matrix with $a_{i 1}=0$ for $i=0, \ldots, r$. In the future we assume all $K$-matrices to be normalized in this way.
} 
1.6 Definition [4]. Let $A$ be a $(t, r)$ - $H$-matrix. An $x$-choice for $A$ consists of $x$ elements of each row of $A$ such that

(1.6) The differences of the form $x_{i}-a_{i k}$ resp. $a_{i k}-x_{i}$ (where $i=$ $0, \ldots, r ; x_{i}$ runs over the chosen elements from row $i$ and $a_{i k}$ over the elements of row $i$ distinct from $x_{i}$ ) together contain each nonzero element of $N$ at least twice.

1.7 Lemma. Let $A$ be an $(r, r)-H$-matrix. Then $A$ has a 1-choice.

Proof. [4, 5.7].

\section{Partitions of $H$-matrices.}

2.1 Definition. Let $A$ be a $(t, r)$ - $H$-matrix. An $x$-partition for $A$ consists of partitions $\mathscr{P}_{i}=\left\{P_{i 1}, \ldots, P_{i x}\right\}(i=0, \ldots, r)$ of the rows of $A$ into $x$ subsets each, satisfying

(2.1) Each nonzero element of $N$ occurs at least twice as a difference of the type $a_{i k}-a_{i m}$, where $a_{i k}$ and $a_{i m}$ are in distinct members of $\mathcal{P}_{i}$.

Partitions for $H$-matrices are in fact generalizations of choices:

2.2 Proposition. Let $A$ be a $(t, r)$-H-matrix. An $x$-choice for $A$ is in fact an $(x+1)$-partition for $A$.

PRoof. Let w.l.o.g. be $a_{i 1}, \ldots, a_{i x}$ the elements of the $x$-choice coming from row $i$ of. $A$. Then define $\mathscr{P}_{i}:=\left\{\left\{a_{i 1}\right\}, \ldots,\left\{a_{i x}\right\},\left\{a_{i, x+1}, \ldots, a_{i t}\right\}\right\}$; this obviously is an $(x+1)$-partition for $A$.

2.3 COROLlaRy. Let $r$ be a prime power. Then there exists an $(r, r)$-H-matrix with a 2-partition.

Proof. By 1.5, 2.2 and 1.7.

We next want to exhibit $\left(r^{n-1}, r\right)-H$-matrices with $\left(r^{n-2}+1\right)$-partitions. To this end, we show

2.4 THEOREM. Let $A$ be an $\left(r^{n-1}, r\right)$-H-matrix (over $\left.N\right)$ that is balanced of type $n$. Then $A$ has an $\left(r^{n-2}+1\right)$-partition $(n \geqslant 2)$.

Proof. The case $n=2$ has already been demonstrated in 2.3. We now assume $n>2$.

Let $A^{\prime}$ be the matrix whose $i$ th row consists of all elements of row $i$ of $A$ that are in $U:=U_{n-1}$ (as in 1.4), i.e. whose $i$ th row is $T_{i 1}^{n-1}$ (using $a_{i 1}=0$ and the notation in (1.5)). Note that by (1.5) we obtain an $(r+1) \times r$-matrix (using 1.5, we have $q_{n}=r$ ) over $U$ and that $U$ has order $r^{2}$ by (1.4). We assert that $A^{\prime}$ is a $K$-matrix. Thus consider any two distinct rows of $A^{\prime}$. The differences arising are pairwise distinct, as $A$ satisfies (1.1) and as $A^{\prime}$ is a submatrix of $A$. For reasons of cardinality, we obtain each element of $U$. Hence $A^{\prime}$ is indeed an $(r, r)-K$-matrix; thus it is actually an $H$-matrix with a 
2-partition, such that each row of $A^{\prime}$ is a subgroup of $U$ (by 1.3, 1.7 and 2.2). Hence $V_{i}:=T_{i 1}^{n-1}$ is a subgroup of $U$ for $i=0, \ldots, r$. Now let $\mathscr{P}_{0}^{\prime}, \ldots, \mathcal{P}_{r}^{\prime}$ be a 2-partition for $A^{\prime}$. Let $\mathscr{P}_{i}$ consist of the two members of $\mathcal{P}_{i}^{\prime}$ and of the sets $T_{i k}^{n-1} \neq T_{i 1}^{n-1}\left(=V_{i}\right)$, for $i=0, \ldots, r$. We assert that $\mathscr{P}_{0}, \ldots, \mathscr{P}_{r}$ is an $\left(r^{n-2}+1\right)$-partition for $A$.

Thus let $z$ be any nonzero element of $N$. If $z$ is actually in $U, z$ occurs at least twice as a difference from distinct members of some $\mathcal{P}_{i}^{\prime}$, hence at least twice from $\mathscr{P}_{i}$.

Finally, assume $z \notin U$. Let $z=a_{i k}-a_{i m}$ be any difference representation of $z$ from $A$. Then we have (by the definition in (1.5)) $T_{i k}^{n-1} \neq T_{i m}^{n-1}$; thus $a_{i k}$ and $a_{i m}$ are in distinct members of $\mathscr{P}_{i}$ (note that in fact $V_{i}=T_{i 1}^{n-1}$ has even been split into two subsets). As this holds for any difference representation of $z,(2.1)$ is obviously satisfied.

Finally each $\mathscr{P}_{i}$ consists of $r^{n-2}+1$ members by (1.5); hence the $\mathscr{P}_{i}$ indeed are an $\left(r^{n-2}+1\right)$-partition for $A$.

2.5 COROLLARY. For each prime power $r$, there is an $\left(r^{n-1}, r\right)-H$-matrix with an $\left(r^{n-2}+1\right)$-partition $(n \geqslant 2)$.

Proof. By 2.4 and 1.5 .

We now prove a fundamental composition theorem:

2.6 THEOREM. Assume the existence of $a(t, r)-H$-matrix with an $x$-partition and of an (s, q)-H-matrix with a y-partition. Assume furthermore

$$
x(r+1) \leqslant q+1 \leqslant t(r+1) .
$$

Then there also is an (st, r)-H-matrix with an $x y$-partition.

We remark that this generalizes and improves $[4,5.3]$.

Proof. Let $A=\left(a_{i k}\right)(i=0, \ldots, r ; k=1, \ldots, t)$ be a $(t, r)$ - $H$-matrix over $M$ with $x$-partition $\mathscr{P}_{0}, \ldots, \mathscr{P}_{r}$, where $\mathscr{P}_{i}=\left\{P_{i 1}, \ldots, P_{i x}\right\}$. Let $B=$ $\left(b_{j m}\right)$ be an $(s, q)$ - $H$-matrix $(j=0, \ldots, q ; m=1, \ldots, s)$ over $N$ with $y$-partition $\mathscr{2}_{0}, \ldots, \mathscr{Q}_{q}$, where $\mathscr{Q}_{j}=\left\{Q_{j 1}, \ldots, Q_{j y}\right\}$. We will construct an (st, $r$ )- $H$-matrix $C$ over $M \oplus N$.

Let $\mathcal{S}$ denote the set of rows of $B$. Partition $\mathcal{S}$ into $r+1$ subsets $S_{0}, \ldots, S_{r}$ satisfying

$$
x<\operatorname{card} S_{i} \leqslant t \text { for } i=0, \ldots, r,
$$

which is possible by (2.2).

Now choose a mapping $F:\{(i, k): i=0, \ldots, r ; k=1, \ldots, t\} \rightarrow \delta$ satisfying (denote $F(i, k)$ by $F_{i k}$ )

(2.4) $F$ is surjective;

(2.5) $F_{i k} \in S_{i}$ for all $(i, k)$; 
(2.6) $F_{i k} \neq F_{i m}$ whenever $a_{i k}$ and $a_{i m}$ are in distinct members of $\mathscr{P}_{i}$; this is possible by (2.3).

Now let $C$ be the $(r+1) \times s t$-matrix over $M \oplus N$ whose $i$ th row is the union of the sets $C_{i k}:=\left\{a_{i k}\right\} \times F_{i k}, k=1, \ldots, t(i=0, \ldots, r){ }^{3}$ We first show that $C$ is a $K$-matrix. Thus consider any two distinct rows of $C$, say row $i$ and $j$. Let $(m, n)$ be any element of $M \oplus N$. As $A$ is a $K$-matrix, there are elements $a_{i k}$ and $a_{j h}$ with $m=a_{i k}-a_{j h}$. As $B$ is a $K$-matrix, the distinct rows $F_{i k}$ and $F_{j h}$ of $B$ satisfy (1.1), i.e. $F_{i k}-F_{j h}=N$. Hence in particular $n$ occurs among these differences. But then clearly $(m, n)$ occurs in $C_{i k}-C_{j h}$. Thus $C$ satisfies (1.1) and is a $K$-matrix.

We now assert that $C$ is in fact an $H$-matrix with an $x y$-partition. Let $R_{i}$ $:=\left\{R_{i j k}: j=1, \ldots, x ; k=1, \ldots, y\right\}$ where $R_{i j k}$ is the union of all sets $\left\{a_{i m}\right\} \times Q_{f(i, m), k}$ with $a_{i m} \in P_{i j}$ where $F_{i m}$ is row $f(i, m)$ of $B$. We will prove that $\Re_{0}, \ldots, \Re_{r}$ is indeed an $x y$-partition for $C$, thus simultaneously proving that $C$ is an $H$-matrix. It is easily seen that $\mathscr{R}_{i}$ is a partition of row $i$ of $C$ containing $x y$ members. Thus we only have to check condition (2.1).

We first consider elements $(m, n)$ of $M \oplus N$ with $m \neq 0$. As $\mathscr{P}_{0}, \ldots, \mathscr{P}_{r}$ is an $x$-partition of $A, m$ occurs at least twice as a difference of the form $a_{i h}-a_{i j}$, where $a_{i h}$ and $a_{i j}$ are in distinct members of $\mathscr{P}_{i}$. By (2.6), we have $F_{i h} \neq F_{i j}$. As $B$ is a $K$-matrix, the difference $F_{i h}-F_{i j}$ of the distinct rows $F_{i h}$, $F_{i j}$ of $B$ contains each element of $N$, in particular $n$. Hence $C_{i h}-C_{i j}$ contains the difference $(m, n)$. Clearly this is a representation of the desired type, as elements with first coordinates in distinct members of $\mathcal{P}_{i}$ by our construction are in distinct members of $\Re_{i}$.

Now consider elements $(0, n)$ with $n \neq 0$. As $\mathscr{2}_{0}, \ldots, \mathscr{2}_{q}$ is a $y$-partition for $B, n$ occurs at least twice as a difference of the form $b_{j h}-b_{j k}$, where $b_{j h}$ and $b_{j k}$ are in distinct members of $2_{j}$. By (2.4), there is a pair $(f, g)$ which is mapped onto row $j$ of $B$. But then $(0, n)$ occurs as difference from $\left\{a_{f g}\right\} \times Q_{j u}$ and $\left\{a_{f g}\right\} \times Q_{j v}$ (where $b_{j h} \in Q_{j u}$ and $b_{j k} \in Q_{j v}$ ). Clearly this is a difference representation of the desired type by our construction of $\mathfrak{R}_{f}$. This proves the theorem.

3. Applications. We will now apply 2.6 together with 2.4 to obtain infinitely many new invariants of regular Hjelmslev planes (using 1.2).

3.1 THEOREM. Let $q$ and $r$ be prime powers satisfying

$$
\left(r^{n-1}+1\right)(r+1) \leqslant q+1<r^{n}(r+1) \quad(n>1) .
$$

Then there exists a $\left(q^{k} r^{n}, r\right)$-H-matrix with an $\left(r^{n-1}+1\right)\left(q^{k-1}+1\right)$-partition for all $k \geqslant 1$.

${ }^{3}$ Note that permuting the elements of the rows of some $H$-matrix again yields an $H$-matrix. Thus the only relevant information on row $i$ is given by the set of elements in that row, not by their ordering. 
Proof. By 2.5 and 2.6 .

3.2 COROLLARY. Let $q$ and $r$ be prime powers satisfying (3.1). Then $\left(q^{k} r^{n}, r\right)$ $\in R H$ (where $R H$ denotes the spectrum of invariants of regular $H$-planes) for all $k \geqslant 1$.

Note that 3.1 is a generalization and improvement of $[4,5.8]$, where we considered the case $n=1$ for choices instead of partitions. Though it does not yield new parameter pairs immediately (for $n=1$ ), it gives much lower partition sizes in the 'product matrix' than the old theorem (applying 2.2). Hence repeated application of 2.6 will give new invariants, as we will point out below in 3.6. We first give some numerical examples.

\subsection{EXAMPLES.}

$\begin{array}{lcccc}r & 2 & & \\ n & 1 & 2 & 3 \\ q & 5^{k} & y(y=8,9,11) & y(y=16,17,19,23) \\ t & 2 \cdot 5^{k} & 4 \cdot y^{k} & 8 \cdot y^{k} \\ x & 2\left(5^{k-1}+1\right) & 3\left(y^{k-1}+1\right) & 5\left(y^{k-1}+1\right) & \text { etc. } \\ r & 3 & 2 & \\ n & 1 & 9 y^{k} & \\ q & y(y=7,8,9,11) y(y=16,17,19,23,25,27,29,31,32) & \\ t & 3 y^{k} & 4\left(y^{k-1}+1\right) & \\ x & 2\left(y^{k-1}+1\right) & & \end{array}$

Here we denote by $t$ the first invariant of the resulting matrix and by $x$ the size of the resulting partition.

3.4 THEOREM. Let $q$ and $r$ be prime powers satisfying (3.1). Let $q^{\prime}$ be a further prime power satisfying

$$
\left(r^{n-1}+1\right)\left(q^{k-1}+1\right)(r+1) \leqslant q^{\prime}+1 \leqslant q^{k} r^{n}(r+1),
$$

where $n, k \geqslant 1$. Then there exists a $\left(q^{\prime m} q^{k} r^{n}, r\right)$-H-matrix with an $\left(r^{n-1}+\right.$ 1) $\left(q^{k-1}+1\right)\left(q^{\prime m-1}+1\right)$-partition for all $m \geqslant 1$.

Proof. By 3.1 and 2.6, using 2.5.

This generalizes and (in case $n=1$ ) improves $[4,5.11]$ for $s=q^{k}$.

3.5 COROLlaRY. Let $q, r, q^{\prime}$ be prime powers satisfying (3.1) and (3.2). Then $\left(q^{\prime m} q^{k} r^{n}, r\right) \in R H$ for all $m \geqslant 1$.

3.6 EXAMPLEs. (i) Let $r=3, q=8, n=k=1$. By 3.5, we obtain $\left(24 q^{\prime m}, 3\right)$ $\in R H$ for $q^{\prime}$ a prime power with $16 \leqslant q^{\prime}+1 \leqslant 96$. The results in [4] only yield the corresponding values for $18 \leqslant q^{\prime}+1 \leqslant 72$ (which are the values that can be obtained as invariants of some (not necessarily regular) $H$-plane by the results of [3]).

(ii) Let us now consider the case of $n=k=1$ for arbitrary $q, r$. Then (3.2) reduces to 


$$
4(r+1) \leqslant q^{\prime}+1 \leqslant q r(r+1) ;
$$

the results in $[4]$ yield by twice applying $[4,5.8]$

$$
2(q+1) \leqslant q^{\prime}+1 \leqslant q(q+1)
$$

(which, by the way, are precisely the values also obtained in [3] as the invariants of some $H$-plane) and by $[4,5.11]$

$$
(q \cdot \rho q)(r+1) \leqslant q^{\prime}+1 \leqslant q r(r+1)
$$

where $\rho q$ denotes the smallest integer $\geqslant(q+1) /(r+1)$.

The lower bound in (3.3) is obviously always much better than the one in (3.5); also, (3.5) tends to include only a few values for $q^{\prime}$ or none at all, as $q$ approaches its upper bound (which is $r(r+1)-1$ by (3.1)). Nevertheless, already (3.5) gave new invariants of $H$-planes (cf. [4, 5.13]).

Let us now discuss the merits of (3.3) and (3.4). The lower bound in (3.3) is always better, if $q \neq 2 r+1,2 r+2$, which are the two lowest possible values for $q$ by (3.1). The upper bound in (3.3) will always be better, if $q \neq r(r+1)$ -1 , which is the biggest possible value for $q$ by (3.1). So, in general, we will in most cases obtain new invariants of $H$-planes (even of regular $H$-planes).

(iii) Now let $r=2, n=3, q=19, k=1$. By 3.5 , we obtain $\left(152 q^{\prime}, 2\right) \in$ $R H$ for prime powers $q^{\prime}$ with $29 \leqslant q^{\prime} \leqslant 455$. The results of [2] and [3] combined allow us only to find the corresponding pairs for $39 \leqslant q^{\prime} \leqslant 379$ as the invariants of some $H$-plane. Again, it is easily seen that the results here will give many new invariants.

It is now obvious how to proceed by using 3.4 and 2.5 with 2.6 etc. recursively. In any case, we will obtain new parameter pairs for $H$-planes in general (and in $R H$ in particular). Nevertheless, using the results obtained up to now it is not possible to construct an $H$-matrix for each known invariant pair, as the results of [2] are stronger than 3.1 (the inequality there gives a better lower bound than (3.1)). However, repeated application of 2.6 also improves lower bounds in many cases (which seems to be more interesting and more difficult than to improve upper bounds).

We will conclude this section by exhibiting all known invariants $(t, r)$ for $H$-planes with $r=2$ or $r=3$ and $t \leqslant 1000$.

3.7 EXAMPLEs. (i) We first consider pairs $(t, 2)$ with $t \leqslant 1000$ that are the invariants of some $H$-plane. According to the list in [2], these were:

(a) the 10 values $t=2^{k}, k=0, \ldots, 9$ (well known, e.g. from $H$-planes constructed from $H$-rings);

(b) the 15 values $2 \cdot 5^{k}(k=1,2,3) ; 4 \cdot 11^{k}(k=1,2) ; 8 \cdot 23$ and $16 \cdot 47$ (by $[3,4.1,5.1])$ resp. $2 \cdot 5 \cdot x(x=11,13,16,17,19,23,27,29)$ (by $[3,6.1])$;

(c) the 16 values $4 \cdot 9,4 \cdot 9^{2}, 8 \cdot 17,8 \cdot 19,16 x \quad(x=$ 
$23,25,27,29,31,37,41,43)$ and $36 x(x=19,23,25,27)$ (by combining [2] and [3]).

Note that [4] does not yield any new information. We now find 10 new values for $t$. By 3.1, there exist $(4 x, 2)-H$-matrices with a 6-partition for $x=8,9,11$ (cf. 3.3). Applying 2.6 and 3.1 we thus obtain the values $32 x$ $(x=17,19,23,25,27,29,31) ; 36 \cdot 17 ; 44 x(x=17,19)$. Thus by now, $(t, 2)-H$-planes are known to exist for 51 values of $t \leqslant 1000$. Note, that all the invariants exhibited are in fact in $R H$ by the results of this paper, except for $16 \cdot 23$ and $16 \cdot 25$.

(ii) Now consider pairs $(t, 3)$ with $t<1000$. By the work of [2] and [3], we know the values

(a) $t=3^{k}, k=0, \ldots, 6$;

(b) $3 \cdot x^{k}, k=1,2, x=7,8,11 ; 21 q, q=16,17,19,23,25,27,29,31$, $32,37,39,41,43,47 ; 24 q, q=17,19,23,25,27,29,31,37,39,41 ; 27 q$, $q=19,23,25,29,31,32,37 ; 33 q, q=23,25,27,29$;

(c) $9 q, q=16,17,19,23,25,29,31,32$;

that are 56 values. By 3.1, we obtain $(3 q, 3)-H$-matrices with a 4-partition for $q=7,8,9,11$; those give (because of the better inequality (3.3)) the 6 new values $24 \cdot 16,27 x(x=16,17)$ and $33 x(x=16,17,19)$.

4. Improving lower bounds. In this last section we will use 2.3 and 2.6 (and sometimes 2.5$)$ to obtain $\left(r^{n}, r\right)$ - $H$-matrices admitting much smaller partition sizes than $r^{n-1}+1$. In this way we will obtain a composition theorem similar to 3.1, but with much improved lower bounds. We thus obtain most of the still missing results of [2] for regular $H$-planes; in fact, in most cases this even improves the lower bounds of [2].

We point out that the $\left(r^{n}, r\right)-H$-matrices to be constructed in this section are not balanced; hence they will not correspond to $n$-uniform $H$-planes by $[5,2.12]$, since all $n$-uniform $H$-planes are balanced. In fact, in the first step in the proof of 4.1 we construct $\left(r^{3}, r\right)$ - $H$-matrices. The corresponding $H$-planes are of type 3 (not of type 4 ); by $[5,3.5]$ they cannot be balanced.

4.1 Theorem. Let $2^{n-1}<x<2^{n}$ and let $r$ be a prime power $\neq 2$. If $x \neq 2,4,5,8$, there always exists an $\left(r^{x}, r\right)$-H-matrix with a $2^{n}$-partition. If $x=5,8$ and $r \geqslant 5$, then there also exists an $\left(r^{x}, r\right)$-H-matrix with a $2^{n}$-partition.

Proof. The assertion holds for $x=1$ by 2.3. By 3.1, there are ( $q r, r)-H$ matrices with a 4-partition for all prime powers $q$ with $2(r+1) \leqslant q+1 \leqslant$ $r(r+1)$. As $r \neq 2$, we can choose $q:=r^{2}$. This settles $x=3$.

By 2.6 and 2.4, we know, that there are $\left(q r^{3}, r\right)-H$-matrices with an 8-partition for all prime powers $q$ satisfying $4(r+1) \leqslant q+1 \leqslant r^{3}(r+1)$; as 
$r \neq 2$, we can always choose $q$ to be $r^{3}$ or $r^{4}$; if $r \geqslant 5$, we can also choose $q$ to be $r^{2}$. This settles $x=5,6,7$.

Again by 2.6 and 2.4 , there are $\left(q r^{k}, r\right)$ - $H$-matrices with a 16-partition $(k=6,7)$ whenever $8(r+1) \leqslant q+1 \leqslant r^{k}(r+1)$; we can always take $r^{4}, \ldots, r^{k+1}$ for $q$, which yields the assertion for $x=10, \ldots, 15$. If $r \geqslant 4$, we can also take $q=r^{3}$, which will yield the desired result for $x=9$, unless $r=3$. But there is a $\left(3^{2}, 3\right)-H$-matrix with a 4 -partition by 2.5 ; using 2.6 , we can construct from this a $\left(3^{5}, 3\right)-H$-matrix with a 8 -partition and then, as above, a $\left(3^{9}, 3\right)$ - $H$-matrix with a 16-partition. If $r \geqslant 5$, we can use the arguments advanced above with $k=5$ and obtain $\left(r^{8}, r\right)$ - $H$-matrices with a 16-partition for these values, too. This is the assertion for $x=8$.

Once more using 2.6 and 2.4, we obtain $\left(q r^{k}, r\right)$ - $H$-matrices with a 32-partition for $k=9, \ldots, 15$ and prime powers $q$ satisfying $16(r+1)<q+1<$ $r^{k}(r+1)$. It is now always possible to choose $q$ to be $r^{6}, \ldots, r^{k+1}$, which yields the assertion for $n=5$. It is now easy to prove the theorem in general by using 2.4 and 2.6 and induction on $n(n \geqslant 5)$.

4.2 Theorem. Let $2^{n-1} \leqslant x<2^{n}$ and $x \neq 6$, 8. Then there exists a $\left(2^{x}, 2\right)$-H-matrix with a $5 \cdot 2^{n-2}$-partition.

Proof. The proof uses the same techniques as that of 4.1 ; hence we merely sketch the steps required and leave the details to the reader. There are $(2,2)-$, $\left(2^{2}, 2\right)-,\left(2^{3}, 2\right)$ - and $\left(2^{4}, 2\right)-H$-matrices with 2-, 3-, 5- resp. 9-partitions by 2.5 . From these construct $\left(2^{5}, 2\right)-,\left(2^{7}, 2\right)-,\left(2^{9}, 2\right)-H$-matrices with 6-, 10-, resp. 18-partitions. From those obtain $\left(2^{h}, 2\right)-(h=10,11)$ and $\left(2^{k}, 2\right)-H$-matrices $(k=12, \ldots, 15)$ with 12 - resp. 20-partitions. Then use induction on $n$ $(n \geqslant 4)$.

We finally give some partitions for all the missing values:

4.3 LEMMA. Let $r$ be a prime power. Then there are $\left(r^{2}, r\right)$ - and $\left(r^{4}, r\right)-H$ matrices with an $(r+1)$ - resp. $\left(r^{3}+1\right)$-partition. There is a $\left(2^{6}, 2\right)$-H-matrix with a 33-partition. There are $\left(3^{5}, 3\right)$ - and $\left(3^{8}, 3\right)$ - $H$-matrices with an 8- resp. $\left(3^{7}+1\right)$-partition. There are $\left(4^{5}, 4\right)$ - and $\left(4^{8}, 4\right)$ - $H$-matrices with 10 - resp. 20-partitions.

Proof. Most of these results are true by 2.5 ; the others by 2.5 and 2.6. We leave the details to the reader.

Using these results, we define a function $f(r, x)$ for prime powers $r$ and natural numbers $x$ by

$$
\begin{aligned}
& f(2,6)=33 ; f(2,8)=129 ; \quad f(2,1)=2 ; \\
& f(2, x)=5 \cdot 2^{n-2} \text { otherwise, where } 2^{n-1} \leqslant x<2^{n},
\end{aligned}
$$

and for $r \neq 2$ by 


$$
\begin{aligned}
& f(r, 2)=r+1 ; \quad f(r, 4)=r^{3}+1 ; \quad f(3,8)=3^{7}+1 ; \\
& f(4,5)=10 ; \quad f(4,8)=20 ; \\
& f(r, x)=2^{n} \quad \text { otherwise, when } 2^{n-1} \leqslant x<2^{n} .
\end{aligned}
$$

We are now ready to state the following improved version of 3.1 .

4.4 THEOREM. Let $r$ be a prime power, $x$ a natural number and assume the existence of an $(s, q)$-H-matrix with a c-partition, where

$$
f(r, x)(r+1) \leqslant q+1 \leqslant r^{x}(r+1) .
$$

Then there exists an $\left(s r^{x}, r\right)$-H-matrix with a $c \cdot f(r, x)$-partition. In particular, this is always true for $s=q^{y}$ and $c=f(q, y)$.

Proof. By 2.6, using 4.1 to 4.3.

4.5 COROLlaRY. Let $r, q$ be prime powers and $x, y$ natural numbers satisfying (4.3). Then $\left(q^{y} r^{x}, r\right) \in R H$.

It is obvious that the lower bound in (4.3) is much better than the one given by (3.1). We finally want to compare (4.3) to the results in [2]. In [2], the same upper bound is obtained; but the lower bound is given by $g(r, x)(r+1)$, where

$$
g(r, x)= \begin{cases}1+r+r^{2}+\cdots+r^{b} & \text { for } x=2 b, \\ 1+r+r^{2}+\cdots+r^{b-1}+2 r^{b} & \text { for } x=2 b+1 .\end{cases}
$$

E.g., if $r=5$ and $x=3$, (4.3) gives the lower bound 24 and (4.4) 66; if $r=3$ and $n=6$, (4.4) yields 160 and (4.3) yields 32 . In general, it is easily seen that the bound in (4.3) is always at least as good as that in (4.4), except for $x=4$ and a couple of exceptional cases; in fact, in most cases it is much better. Note that asymptotically $f(r, x)$ is $\log _{2} x$, while $g(r, x)$ is $r^{x / 2}$.

Clearly, this also improves the results of repeated use of 2.6; for instance, the comparitively weak inequality (3.2) could now be improved to the lower bound $f(r, n) f(q, k)$. Hence' we have again obtained a great many new invariants for (even regular) $H$-planes.

\section{REFERENCES}

1. P. Dembowski, Finite geometries, Springer-Verlag, Berlin and New York, 1968.

2. D. A. Drake, More new integer pairs for finite Hjelmslev planes, Illinois J. Math. 19 (1975), 618-627.

3. D. A. Drake and H. Lenz, Finite Klingenberg planes, Abh. Math. Sem. Univ. Hamburg 44 (1975), 70-83.

4. D. Jungnickel, Regular Hjelmslev planes, J. Combinatorial Theory (to appear).

5. __ On balanced regular Hjelmslev planes, Geometriae Dedicata (to appear).

6. G. Törner, Über den Stufenaufbau von Hjelmslev-Ebenen, Manuscript (1976).

Department of Mathematics, University of Florida, Gainesville, Florida 32611

Current address: Institut für Mathematik II, Freie Universität Berlin, FB19, WE2, 1000 Berlin 33, Königin-Luise-Strasse 24/26, Berlin, Federal Republic of Germany 